\title{
Frequency Standards Based on Stored Ions
}

DAVID I. WINELAND

Invited Paper
The state of development of frequency standards based on stored ions is reviewed Several preliminary demonstrations of the concept have already shown a level of performance approaching that of today's cesium-beam standards (accuracy of one part in 103). The potential for accurately measuring or reducing all known svstematic effects suggests that frequency standards based on stored ions with inaccuracies of one part in $10^{15}$ are obtainable and eventually they could be orders of magnitude better than this. This performance is a result of extremely high-Q resonances (e.g., millihertz linewidths at microwave trequencies) and a very small second-order Doppler shift which follows with the addition of techniques for ion cooling

\section{INTRODUCTION}

For many years, it has been realized that the technique of ion storage provides the basis for spectroscopy of very-high resolution and accuracy. This notion is based on the fact that atomic or molecular ions can be stored for long periods of time without the usual perturbations associated with confinement. First, samples of ions can be stored using electromagnetic fields in "traps" for times as long as days [1]. [2]. In practice this has two important advantages: 1) Spectral resolution is not limited by "transit-time" effects such as the length of time it takes cesium atoms to traverse the region between the two ends of the Ramsey cavity [3] Such transit-time broadening is, of course, a direct manifestation of Heisenberg's uncertainty relation for time and energy. In the case of stored ions, linewidths of about 0.01 $\mathrm{Hz}$ have already been observed [4] (see Fig. 1). This would correspond to a cesium-beam tube of about $10-\mathrm{km}$ length! (neglecting, of course, the effects of gravity). 2) Since the ions are stored, their average velocity $\langle\vec{v}\rangle$ approaches zero; in practice this means that first-order Doppler effects can be extremely small [5]. This characteristic, which is also shared by rubidium and hydrogen maser clocks, gives an advantage over atomic beam devices where a correction

Manuscript received November 19, 1984; revised August 14, 1985 The preparation of this manuscript and the ion storage work at NBS, Boulder, were supported in part by the U.S. Otfice of Naval Research and the U.S. Airforce Office of Scientific Research.

The author is with Time and Frequency Division, National Bureau of Standards, Boulder, CO 80303, USA.

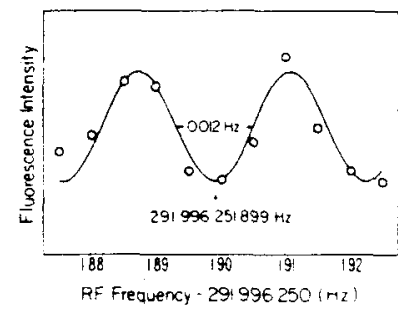

Fig. 1. Graph of a hyperfine resonance of trapped ${ }^{25} \mathrm{Mg}$. ions. The oscillatory lineshape results from the use of the Ramsey resonance method, implemented by applying two coherent RF pulses $1.02 \mathrm{~s}$ long, separated by $41.4 \mathrm{~s}$. The solid curve is a theoretical lineshape (from [4]).

must be made for cavity phase shift errors-a form of residual first-order Doppler effect.

An additional advantage of the stored ion technique is that ions can be confined without the usual perturbations associated with confinement - for example, the frequency shifts associated with collisions of atoms with like atoms, buffer gasses, or container walls such as in the rubidium or hydrogen maser clocks. Ions are often stored under ultrahigh vacuum conditions so that frequency shifts due to collisions with background neutrals can be made negligible. Frequency shifts due to ion-ion collisions are caused by the electric fields of the Coulomb repulsion. One is concerned with quadratic Stark shifts (i.e., $\delta v \propto\left\langle E^{2}\right\rangle$ ) and in many cases these shifts can be extremely small [5]-[7].

Perhaps the main disadvantage of the stored ion technique is that the number of ions that can be stored is rather small. Typical densities are approximately $10^{6} / \mathrm{cm}^{3}$ and trap volumes about $1-10 \mathrm{~cm}^{3}$ so that maximum numbers are on the order of $10^{6}$ or less. In some cases, however, it is possible to detect atomic transitions with 100 -percent efficiency [2], [8], in this case, the signal-to-noise ratio need only be limited by the number of ions which make the transition [8]; for $10^{6}$ ions this can give a signal-to-noise ratio approaching $10^{3}$. We note that if we could obtain much higher densities by using larger confining fields then we would lose one of the advantages of the technique 
because electric-field frequency shifts would become troublesome in very-high-resolution work.

\section{Trapped lons}

Storage of ions for spectroscopy has principally been accomplished in four types of "traps": the RF or Paul trap, the Penning trap, the Kingdon (electrostatic) trap, and the magnetostatic trap ("magnetic bottle") [1], [2]. Magnetic bottles have had limited use in high-resolution work because the trapping relies on spatially inhomogeneous magnetic fields which can cause shifts and broadening on magnetic-field-dependent lines. The Kingdon trap [9] is perhaps the simplest using only static electric fields for trapping. Since an electrostatic potential minimum cannot exist in a charge-free region, the Kingdon trap relies on dynamical equilibrium for trapping (ions orbit around an attractive wire). The Kingdon traps have been used in spectroscopic experiments by Prior and his colleagues [10] but so far have not been applied to atomic clocks.

The Paul [17] or RF trap uses inhomogeneous RF electric fields to provide confinement in a pseudopotential well [1], [2]. It is the three-dimensional analog of the Paul quadrupole mass filter. To see how it works we first note that in a (homogeneous) sinusoidal RF electric field, ion motion is sinusoidal but is $180^{\circ}$ out of phase with respect to the electric force. If the field is somewhat inhomogeneous, it is easy to show that the force on the ion averaged over one cycle of the driven motion is towards the region of weaker field [1]. Since an electric field minimum can exist in a charge-free region, stable trapping can be accomplished. Such a trap is shown schematically in Fig. 2 where the three trap electrodes are shaped to provide an electric potential of the form $\left(r^{2}-2 z^{2}\right)$ inside the trap. For this "ideal" trap

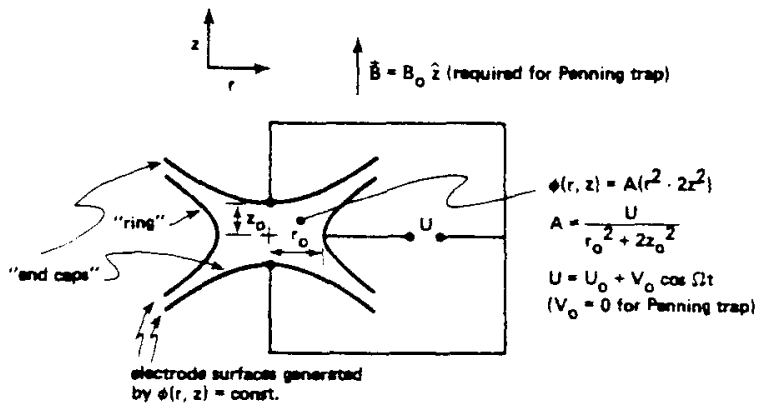

Fig. 2. Schematic representation of the electrode configuration for the "ideal" Paul (RF) or Penning trap. Electrode surfaces are figures of revolution about the $z$ axis and are equipotentials of $\phi(r, z)=A\left(r^{2}-2 z^{2}\right)$. (Cylindrical coordinates are used with the origin at the center of the trap.) Typical dimensions are $\sqrt{2} z_{0}=r_{0} \equiv 1 \mathrm{~cm}$. Typical operating parameters are for the Paul trap, $V_{0} \cong 300 \vee / \mathrm{cm}, \Omega / 2 \pi \cong$ $1 \mathrm{MHz}$. For the Penning $\operatorname{trap} V_{0} \equiv 1 \mathrm{~V}, B \equiv 1 \mathrm{~T}$.

shape, an ion is bound in a nearly harmonic well; detection of the ion-well frequencies can then be used to perform mass analysis

The "ideal" Penning [12] trap uses the same electrode configuration as in Fig. 2 but uses static electric and magnetic fields. A harmonic potential well is provided along the " $z$ " axis by static electric fields. This, however, results in a radial electric field which forces the ions towards the "ring" electrode. This effect can be overcome if a static magnetic field $\vec{B}$ is superimposed along the " $z$ " axis. In this case, the $x-y$ motion of the ions is a composite of circular cyclotron orbits (primarily due to the $\vec{B}$ field) and a circular $\vec{E} \times \vec{B}$ drift ("magnetron" motion) about the trap axis.

\section{Stored ion Spectra and Applications to Clocks}

The application of stored ion spectra to atomic clocks and frequency standards has been pursued with different philosophies in mind. For reasons discussed below; there are fundamental advantages to using lasers in experiments on stored ion spectra, however, if one's goal is to produce field-usable devices with competitive specifications and price, use of lasers is most often precluded. In research and primary standards laboratories, such limitations are less of a concern and the emphasis is on ultimate performance. Therefore, the various experiments discussed below differ in this tradeoff between performance and technical practicability.

The experiments of Dehmelt and collaborators [1] in the 1960 s showed the ability of the stored ion technique for obtaining very-high resolution in atomic spectra. In one of these experiments, the ground state hyperfine transition $\left(\nu_{0} \cong 8.7 \mathrm{GHz}\right)$ in the ${ }^{3} \mathrm{He}^{+}$ion was measured in an RF trap with a linewidth of only $10 \mathrm{~Hz}$ [13], but the accuracy was limited by the second-order Doppler shift $\left(\delta \nu / \nu_{0}=\right.$ $\left.-1 / 2\left\langle v^{2}\right\rangle / c^{2}\right)$ to $10 \mathrm{~Hz}$ ( $c=$ speed of light). This frequency shift is accentuated for many ions stored in an RF trap because of the mechanism of "RF heating" caused by the rather large imposed inhomogeneous RF electric fields required for trapping [1] Fortunately, the cooling mechanisms discussed below can minimize the effects of this heating.

Starting with the work of Major and Werth in 1973 [14], considerable effort has been devoted by different groups [15]-[18] to develop the ${ }^{799} \mathrm{Hg}^{+}$ion in an RF trap as a frequency standard [19]. The group at Orsay [16] has, in fact. made a working frequency standard with short-term stability comparable to that of a commercial cesium-beam frequency standard. Stabilities near $\sigma_{y}(\tau)=10^{12} \tau^{-i / 2}$ should be obtainable [16], [17]. This work on the mercury ion has perhaps been motivated by three considerations: 1) the mercury-ion hyperfine frequency $(\cong 40 \mathrm{CHz})$ is probably the highest of any ion that might be used in a microwave frequency standard. Therefore, if the interaction time is limited by relaxation processes that are independent of the ion, then $\mathrm{Hg}^{+}$ions would have the highest $Q$. 2) Since $\mathrm{Hg}^{-}$ is relatively heavy, the second-order Doppler shift can be fairly small at a given temperature. For example, if the ions are thermalized to room temperature, the second-order Doppler shift due to the "secular" motion in the pseudopotential well [1], [2], [17] amounts to a fractional frequency shift of only about $2 \times 10^{-13}$. 3) A convenient overlap of the optical spectra of ${ }^{199} \mathrm{Hg}^{+}$and ${ }^{202} \mathrm{Hg}^{+}$allows ${ }^{109} \mathrm{Hg}^{-}$to be pumped with a ${ }^{202} \mathrm{Hg}^{+}$lamp, much the same way as pumping is accomplished in a Rb-cell frequency standard.

In these experiments, the second-order Doppler frequency shift can be minimized by cooling the ions with a light neutral buffer gas (e.g., helium or hydrogen). This buffer gas (pressures up to $10^{-2} \mathrm{~Pa}$ ) acts as a viscous medium so that the secular motion of the ions in the pseudopotential well is thermalized to the ambient temperature [1]. Unfortunately, one is left with the second-order Doppler shift due to the energy in the micromotion [1]. [7]. 
This effect is perhaps most easily seen by assuming that the secular motion is completely "frozen." The ion cloud cannot collapse because of the repulsive space-charge fields; therefore, ions on the edge of the "cloud" are still being driven by the RF fields. The energy in this driven motion then gives rise to the limiting second-order Doppler frequency shift [7]. Fortunately, this effect can be modeled fairly well [17], [18] and estimated systematic uncertainties [17] due to this effect should be around $2 \times 10^{-13}$. By introducing the cooling gas, one has given up the immunity from buffer gas shifts but these are estimated [17] to be below $10^{-13}$

In addition, optical pumping experiments on stored ions have been performed using tunable lasers as light sources. The ground-state hyperfine splittings of ${ }^{737} \mathrm{Ba}^{+}[20],{ }^{135} \mathrm{Ba}^{+}$ [21], and ${ }^{177} \mathrm{Yb}^{+}$[22] have been measured, using pulsed dye lasers and RF traps. Microwave resonances as narrow as 60 $\mathrm{mHz}$ were observed in ${ }^{171} \mathrm{Yb}^{+}$. This yields a line $Q$ of $2 \times 10^{11}$. In some cases, optical pumping out of the absorbing ground state prevents use of the double-resonance method. This problem may be overcome, however, with the use of collisional relaxation [20], [23].

Even with viscous drag cooling, one is faced with uncertainties in the second-order Doppler shift if it is desired to improve the accuracy of atomic clocks significantly beyond that of the best laboratory cesium devices. In 1975, proposals were made [24], [25] to get around this general problem of the second-order Doppler shift by a process commonly called laser (or sideband) cooling. In this process, radiation pressure from lasers is used in conjunction with the frequency selectivity provided by the first-order Doppler effect to damp the ion motions to sub-Kelvin temperatures [2]. If these low temperatures can be obtained on $\mathrm{Hg}^{+}$ions, for example, the magnitude of the secondorder Doppler shift would be less than $10^{-15}$.

As a step towards realizing a frequency standard based on laser-cooled stored ions, a clock based on nuclear spin flip hyperfine transitions in ${ }^{9} \mathrm{Be}^{+}$ions has been constructed [26]. The average frequency of an RF oscillator was locked to the $\left(M_{1}, M_{1}\right)=(-3 / 2,1 / 2)$ to $(-1 / 2,1 / 2)$ transition in the ground-state of ${ }^{9} \mathrm{Be}^{+}$, near the magnetic field $(0.8194$ T) at which the first derivative of the frequency with respect to field goes to zero. The ions were stored in a Penning trap and cooled to less than $2 \mathrm{~K}$ by about $20 \mu \mathrm{W}$ of $\mathrm{CW} 313-\mathrm{nm}$ radiation. The $303-\mathrm{MHz}$ resonance was observed with 25 $\mathrm{mHz}$ linewidth by RF-optical double resonance. The frequency stability of the locked oscillator $(\sigma, \tau) \cong 2 \times$ $10^{-11} \tau^{-1,2}$ ) was comparable to that of commercial $C_{5}$ atomic beam frequency standards. The frequency accuracy was approximately $1 \times 10^{-13}$, limited primarily by the uncertainty of the second-order Doppler shift due to heating of the ions during the RF resonance period, when the cooling radiation was shut off in order to avoid light shitts. Although laboratory cesium standards are somewhat better than this, this first experiment is hopefully indicative of the potential for stored ion devices.

Of course, in a frequency standard or clock, measurement imprecision $\left(\delta v_{\text {error }} / v_{0}\right)$ is approximately equal to $(Q \cdot S / N)^{-1}$ where $Q \equiv \nu_{0} / \Delta \nu_{0}$ and $S / N$ is the signal-tonoise ratio for detecting the number of ions that have made the transition. If the ion's radiative linewidth is small enough, then the experimentally observed linewidth (\lrcorner$\left.\nu_{0}\right)$ need only be limited by the length of time taken to induce the transition. Because of this, $\Delta \nu_{0}$ is probably independent of the species of trapped ion used. Theretore, we would like to use as high a frequency $\left(\boldsymbol{\nu}_{0}\right)$ as possible in order to increase $Q$ and reduce measurement imprecision. This is the single disadvantage of $\mathrm{Be}^{+}$ions since the interesting "clock" transitions are only around $300 \mathrm{MHz}\left(Q \geq 10^{10}\right)$. For this reason, a better ion for a laser-cooled microwave clock is perhaps $\mathrm{Hg}^{-}[8]\left(\nu_{0} \cong 40 \mathrm{CHz}\right.$ for $\left.{ }^{199} \mathrm{Hg}^{+}\right)$. Untortunately, laser cooling is much harder to achieve than for $\mathrm{Be}^{+}$(partly because the $194-\mathrm{nm}$ cooling radiation is difficult to produce), and has not been done yet.

\section{iv. Optical Clocks}

A logical extension of this idea is to go to a much higher frequency; for example, to use a narrow optical transition. The anticipated $Q$ in this case can be extremely high, $10^{15}$ or more. A number of transitions in various ions have been proposed [2]; Dehmelt [27] was the first to suggest that such extremely high-resolution spectroscopy could be carried out using single photon transitions in, for example, single group IIIA ions. For instance [27], the ${ }^{1} S_{0} \leftrightarrow{ }^{3} P_{0}$ transition in $\mathrm{TH}^{+}(\lambda=202 \mathrm{~nm})$ has a $Q \equiv 5 \times 10^{14}$. For such single-photon optical transitions, it is desirable to approximately satisfy the Lambe-Dicke criterion (spatial confinement to dimensions less than a wavelength where first-order Doppler broadening is made negligible); this is most easily accomplished with single trapped ions [2], [27]. Others [28] have proposed using two-photon Doppler-free transitions, for example, the ${ }^{2} \mathrm{~S}_{1 / 2} \rightarrow{ }^{2} \mathrm{D}_{5 / 2}$ transition in $\mathrm{Hg}^{+}(\lambda=563 \mathrm{~nm}$, $\left.Q \cong 7 \times 10^{14}\right)$. Two-photon optical transitions using equal frequency photons have the advantage of eliminating the first-order Doppler effect for a cloud of many ions where it is impossible to satisfy the Lambe-Dicke criterion. They ultimately have the disadvantage that the rather large optical fields necessary to drive the transition cause undesirable ac Stark shifts [8], [28].

The projected accuracy for optical frequency standards using single ions is extremely high. As an example, in $\mathrm{In}^{+}$, the linewidth of the ' $S_{0} \rightarrow{ }^{3} P_{1}$ "cooling" transition is about $1.3 \mathrm{MHz}$, this implies a second-order Doppler shift of $10^{-19}$ or lower [2]. Other systematic shifts can occur [1], [2], [4], [6], [8], [14]-[18], [26]-[29] but it is not unreasonable to think they will be controllable to this level. These extreme accuracies make important the problem of measurement imprecision since the signal-to-noise ratio on a single ion will at best be about one for each measurement cycle. Practically speaking, this means that a long averaging time will be required to reach a measurement precision equal to these accuracies. In fact, for a while, the accuracy and resolution may be limited by laser linewidth characteristics (linewidth and linewidth symmetry). However, the potential for extremely narrow lasers also exists [30]. Unfortunately, to use such laser devices as clocks one must count cycles of the radiation, that is, measure its phase. At microwave frequencies this is straightforward. At optical frequencies it is technically feasible but very hard [31].

\section{CONCLUSION}

Compared to the development of the cesium-beam frequency standard, the hydrogen maser and the rubidium frequency standard, clocks based on stored ions, are in their infancy. Several laboratories are actively studying such de- 
vices because it appears that systematic frequency shifts can eventually be measured and controlled to better than 1 part in $10^{15}$. This potential, although not yet realized, suggests that frequency standards and clocks with inaccuracy of one part in $10^{15}$ can be obtained.

\section{REFERENCES .}

[1] H. G. Dehmelt, Adv. Atom. Mol. Phys., vol. 3, p. 53, 1967, and Adv Atom. Mol. Phys., vol. 5, p. 109, 1969.

[2] D. J. Wineland, W. M. Itano, and R. S. Van Dyck Jr., Adv. Atom. Mol Phys, vol. 19 , p 135,1983

D. J. Wineland. W. M. Itano, J. C. Bergquist, J. J. Bollinger, and 1. D. Prestage, in Atomic Physics 9 ,

R. S. Van Dyck Ir. and E. N. Fortson, Eds. Singapore:World Sci. Pub., 1984, p. 3.

[3] N. F. Ramsey, Molecular Beams. London, England: Oxford Univ. Press, 1956

[4] W. M. Itano and D. 1. Wineland, Phys. Rev., vol. A24, p. 1364. 1981.

[5] D. I. Wineland, Science, Vol. 226, p. 395, 1984

[6] W. M. Itano, L. L. Lewis, and D. I. Wineland, Phys. Rev., vol A25, p. 1233, 1982.

[7] D. . Wineland in Precision Measurement and Fundamental Constants II. B. N. Taylor and W. D. Phillips, Eds. Boulder, CO: Nat. Bur. Stand. Spec Publ. 617, 1984, p. 83.

[8] D. . Wineland, W. M. Itano, I. C. Bergquist, and F. L. Walls, in Proc. 35th Annu. Symp. on Frequency Control, 1981, p. 602. (Copies available from Electronic Industries Assoc. 2001 Eye St. N.W., Washington, DC 20006.)

[9] K. H. Kingdon, Phys. Rev, vol. 21, p. 408, 1923

[10] M. H. Prior and E. C. Wang, Phys. Rev., vol. A16, p. 6, 1977.

[11] E. Fischer, Z. Physik, vol. 156, p. 1, 1959

R. F. Wuerker, H. Shelton, and R. V. Langmuir, 1. Appl. Phys. vol. 30, p. 342, 1959.

[12] F. M Penning Physica vol 3, p. 873, 1936

[13] H. A. Schuessler, E. N. Fortson, and H. C. Dehmelt, Phys. Rev. vol. 187, p. 5,1969

[14] F. C. Major and C. Werth, Phys. Rev. Lett., vol. 30, p. 1155. 1973.

[15] M. D. McGuire, R. Petsch, and G. Werth, Phys. Rev, vol. A17, p. 1999,1978

[16] $M$. Jardino, M. Desaintfuscien, R. Barillet, I. Viennet, P. Petit, and C. Audoin, Appl. Phys., vol. 24, p. 107, 1981.

[17] L. S. Cutler, R. P. Giffard, and M. D. MCGuire, in Proc. 37 th Annu. Symp on Frequency Control 1983, p. 32. (Copies available from Systematics Ceneral Corp., Brinley Plaza, Rt. 38 ,
Wall Township, NJ 07719.)

L. S. Cutter, R. P. Giffard, and M. D. McCuire, Appl. Phys., vot B36, p. 137,1985

[18] $M$. Jardino, F. Plumelle, $M$. Desaintfuscien, and I. L. Duchene, in Proc. 38th Annu. Symp. on Frequency Control (Philadelphia, PA, 1984), p. 431. (Copies available from IEEE, 445 Hoes Lane, Piscataway, NJ 08854.)

[19] H. A. Schuessler, Metrologia, vol. 7, p. 103, 1971.

[20] R. Blatt and C. Werth, Phys. Rev., vol. A25, p. 1476, 1982

[21] W. Becker, R. Blatt, and G. Werth, J. Phys. (Orsay, France). vol. 42, p. C8-339, 1981

[22] R. Blatt, H. Schnatz, and G. Werth, Phys. Rev. Lett., vol. 48 P. 1601,1982

[23] W. Ruster, 1. Bonn, P. Peuser, and N. Trautmann, Appl. Phys. vol. B30. p. 83, 1983

[24] T. W. Hänsch and A. L. Schawlow, Opt. Commun., vol. 13, p. 68,1975

[25] D. J. Wineland and H. G. Dehmelt, Bull. Amer. Phys. Soc vol. 20, p. 637, 1975.

[26] I. I. Bollinger, W. M. Itano, and D. J. Wineland, in Proc. 37th Ann. Symp. on Frequency Control, 1983, p. 37. (Copies available from Systematics General Corp. Brinley Plaza Rt 38 Wall Township, N) 07719.)

J. J. Bollinger, J. D. Prestage, W. M. Itano, and D. f. Wineland, Phys. Rev Lett. vol 54, p. 7000,7985

[27] H. Dehmelt, IEEE Trans. Instrum. Meas., vol. IM-31, p. 83 . 1982.

[28] P. L. Bender, J. L. Hall, R. M. Garstang, F. M. J. Pichanick, W. W Smith. R. L. Barger, and I. B. West, Bull. Amer Phws Soc. vol. 27 , p. 599,1976

[29] D. I. Wineland and W. M. Itano, Bull. Amer. Phys. Soc., vol. 27. p. 864,1982

[30] 1. L. Hall, L. Hollberg, Ma Long-Shen, T. Baer, and H. G. Robinson, 1. Phys. (Orsay, France), vol 42, p. C8-59, 1981. A. Yariv and K. Vahala, IEEE J. Quantum Electron., vol. QE-19 p. 889.1983.

R. W. P. Drever I. L. Hall, F. V. Kowalski, I. Hough, G. M Ford A. J. Manley, and H. Ward, Appl. Phys B, vol. 37, p. 97, 1983 1. Hough, D. Hils, M. D. Rayman, L. S. Ma, L. Hollberg, and 1. L. Hall, Appl. Phys. B, vol. 33, p. 179, 1984.

[31] See: Proc. 3rd Symp. On Frequency Standards and Metrology (J. Phys., vol. 42, Collog. (-8), Dec. 1981

V. P. Chebotayev, V. C. Goldort, V. M. Klementyev, M. V Nikitin, B. A. Timchenko, and V. F. Zakharyash, Appl. Phys. B vol. 29, p. $63,1982$.

$D$ A Jennings, $C$. R . Pollock, $F$. R Petersen, R. E Drullinger, K. M. Evenson, I. S. Wells, J. L. Hall, H. P. Layer, Opt. Lett., vol 8, p. $136,1983$.

K. M. Baird Phys Today vol 36 no 1, p. 52, 1983

B. Whitford, Appl Phys. B, vol. 35, p. 119, 1984 